\title{
La ética militar y familia: importancia de las pautas de crianza para la conducta del individuo en sociedad ${ }^{1}$
}

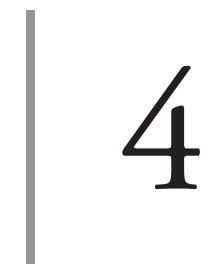

https://doi.org/10.21830/9789585377127.04

\author{
Henry Mauricio Acosta Guzmán ${ }^{2}$ \\ Karen Yuliana Galeano Torres ${ }^{3}$ \\ Mariano Augusto Sánchez Valcárcel ${ }^{4}$ \\ Escuela Superior de Guerra "General Rafael Reyes Prieto"
}

\section{Resumen}

En este capítulo de libro se aborda la relación entre pautas de crianza en el entorno familiar, la conducta en sociedad y la ética profesional desde la teoría conductual, así como el entorno familiar para la formación de la ética profesional. Se trata de un trabajo descriptivo que empleó una metodología cualitativa, consistente en el análisis a través de la revisión teórica, conceptual y coyuntural, que recopiló bibliografía de documentos académicos, investigaciones y trabajos de campo nacionales e internacionales realizados en torno a los conceptos de familia, crianza, ética y moral. En este ejercicio se pudo

1 Este capítulo de libro presenta los resultados colaborativos del proyecto de investigación "Ética militar en entornos complejos de seguridad y defensa: lecturas y aportes desde la experiencia de las Fuerzas Militares de Colombia”, del grupo de investigación Masa Crítica de la Escuela Superior de Guerra "General Rafael Reyes Prieto", categorizado en B por Minciencias y con código de registro COL0123247. Los puntos de vista pertenecen a los autores y no reflejan necesariamente los de las instituciones participantes.

2 Magíster en Seguridad y Defensa Nacionales de la Escuela Superior de Guerra "General Rafael Reyes Prieto". Politólogo de la Universidad Nacional de Colombia. Investigador del Departamento de Ética y Liderazgo. Editor asistente de la Revista Estudios en Seguridad y Defensa e investigador del grupo de investigación Masa Crítica, de la Escuela Superior de Guerra, Colombia. Orcid: https://orcid.org/00000003-4485-8845 - Contacto: henry.acosta@esdegue.edu.co

3 Psicóloga de la Corporación Universitaria Minuto de Dios. Auxiliar de investigación. Orcid: https://orcid.org/0000-0002-8304-8805 - Contacto: kgaleanotor@uniminuto.edu.co

4 Teniente Coronel del Ejército Nacional de Colombia. Especialista en Seguridad y Defensa, Escuela Superior de Guerra "General Rafael Reyes Prieto". Profesional en Ciencias Militares de la Escuela Militar de Cadetes “General José María Córdova”. Jefe del Departamento de Ética y Liderazgo de la Escuela Superior de Guerra "General Rafael Reyes Prieto”, Colombia. Orcid: https://orcid.org/0000-00015785-1816 - Contacto: mariano.sanchez@esdegue.edu.co 
identificar como principales resultados que la formación de líderes éticos es un aspecto importante para las Fuerzas Militares de Colombia porque permite que los individuos tengan un mejor desenvolvimiento en la sociedad y en su quehacer como funcionarios. Si bien la educación en las instituciones militares es el pilar fundamental para la doctrina, se debe precisar que esta también debe inculcarse desde la niñez, en razón a que es una etapa temprana donde la persona establece su perfil motivacional conforme a las pautas de crianza. Es así que como principal conclusión de la investigación se estableció que la responsabilidad en la formación de personas éticas no es solo de las instituciones educativas, sino que también involucra el ámbito familiar como principal escenario de interacción social.

Palabras clave: educación; ética; familia; pautas de crianza.

\section{Introducción}

Existe la inquietud acerca de cómo los procesos psicológicos y la construcción de la conducta del ser humano influyen en el desarrollo ético de los individuos, debido a que la conducta es la respuesta generada del individuo frente a estímulos del entorno, es decir, el individuo es reactivo al escenario social. En este sentido, se entiende la conducta como el último eslabón de un proceso mental que lleva al individuo a actuar de una manera determinada, acciones que generalmente se acondicionan a variables en el momento de la formación del individuo en etapas tempranas, y por ello resultan importantes las pautas de crianza, lo cual guarda relación con las teorías conductuales que explican los patrones de la conducta de un ser humano.

El presente documento indaga acerca del modelo psicológico explicativo del condicionamiento operante propuesto por Burrhus Skinner, en este caso analizado en la familia. Desde esta perspectiva, la conducta del ser humano es aprendida, pues el individuo adquiere conocimientos al comportarse a partir del reforzamiento de su conducta, y el reforzamiento, también llamado el refuerzo positivo y refuerzos negativos, aumenta la probabilidad de influir una conducta (Laurence \& Woodward, 1996). Es así que contemplar castigos, denominados refuerzos negativos, influye en el condicionamiento operante, por ello el núcleo familiar es un componente fundamental en la formación del individuo, en razón a que el reforzamiento permanente de competencias como la disciplina 
impacta a largo plazo en el contexto social y en el profesional, donde también se genera un aprendizaje permanente (Padilla, 2014; Trillo, 2018).

Es por esto que en este trabajo se busca establecer la importancia de fortalecer la conducta desde las pautas de crianza en el entorno familiar, puesto que en la etapa de la infancia es donde más se potencia el aprendizaje. Por ello, se resalta el papel preponderante del entorno familiar, pues en él se imparten acciones que fortalecen el actuar ético, ya que es el núcleo que establece el primer modelo que sigue el individuo en sus etapas tempranas para relacionarse en sociedad (Fabelo, 2001).

$\mathrm{Al}$ respecto, cabe señalar que en el escenario social contemporáneo existe una crisis ética debido al mantenimiento de conductas moralmente incorrectas, por lo cual se considera importante reorientar la formación del individuo hacia la ética, la moral y, en especial, la ética profesional en el caso de Colombia. Precisamente, para dar dirección al documento se planteó el siguiente interrogante: ¿Cómo las conductas del individuo en sociedad y las pautas de crianza en la familia pueden generar un impacto en el desarrollo de la ética profesional?

\section{Las pautas de crianza en el entorno familiar, la conducta en sociedad y la ética profesional desde la teoría conductual}

Con base en las teorías psicológicas del desarrollo moral, se pretende explicar el fenómeno moral en la conducta de un individuo. Por esta razón, a continuación se abordan aproximaciones teóricas y conceptuales que facilitan la comprensión del porqué los seres humanos generan una respuesta conductual frente a determinados escenarios.

Para comenzar se debe afirmar que el individuo responde a estímulos y con ello se presentan actuaciones concretas en las que debe elegir entre lo bueno y lo malo, lo correcto y lo incorrecto. Al respecto, Colón (2004) explica cómo se desarrolla el ethos en el carácter moral que interviene en el comportamiento humano, debido a que este facilita la comprensión de la relación que tiene el comportamiento de un individuo y la moral, o dicho de otra manera, 
son las costumbres, la conducta, la que permite perfilar la moralidad de un individuo (Kaplan \& Sadock's, 1998).

Inicialmente, la moral se entiende como la aprobación de las reglas o normas, basadas en los derechos y deberes de un individuo, que a su vez dirigen su conducta sobre lo que es correcto en determinado contexto o aceptado socialmente. Con esta orientación moral, el individuo aprende a emitir juicios, tomando como base el aprendizaje que ha tenido en la educación escolar o en el entorno familiar, los cuales se rigen por normas y pautas de crianza y tienen un impacto en la toma de conciencia. El individuo por naturaleza posee el libre arbitrio y, de manera crítica y autosuficiente, puede tomar sus propias decisiones con base en su sentido individual, lo cual, a su vez, implica que se comporte de manera diferente y se someta a normas establecidas (Trillo, 2018).

Según Trillo (2018), la ética y la moral aportan al bienestar de los seres humanos y modifican la convivencia entre ellos, debido a que establecen normas de conducta y de relación que se generan en edades tempranas en el entorno familiar y, posteriormente, en sociedad. La formación tiene un sentido que es operativo para el individuo.

Por lo anterior, la teoría del condicionamiento operante permite determinar elementos que condicionan la conducta del ser humano, como el refuerzo positivo o negativo, los cuales se pueden asociar con las pautas de crianza en el entorno de familia, en razón a que ese sistema y organización se encarga de generar condiciones que facilitan el aprendizaje de los individuos. En efecto, el condicionamiento operante hace que el sujeto mantenga, aumente o disminuya una conducta mediante la asociación de consecuencias frente a un estímulo, es decir que el aprendizaje es un "cambio de la conducta ocasionado por la experiencia” (Trillo, 2018, p. 2).

\section{Pautas de crianza a través del condicionamiento operante}

Para modificar la conducta es indispensable promover el cambio a través de técnicas de intervención psicológicas que impacten de manera positiva el comportamiento del individuo con el fin de potenciar sus habilidades y 
que aproveche las oportunidades en un contexto determinado. Es así que la convivencia de manera útil y asertiva es importante $y$, por consiguiente, se deben reforzar las conductas que son apropiadas y disminuir las que no lo son a través de su condicionamiento para consolidarlas. No obstante, en la sociedad con principios conservadores aún existe una tendencia al grito, al golpe, al insulto o a la descalificación como método de educación y corrección de conductas no deseadas, una práctica poco efectiva que fue empleada por educadores o familiares y que, por el contrario, suele ser contraproducente, pues genera frustración, miedo al fracaso, baja autoestima o abandono de las tareas (Trillo, 2018).

En esta medida, se plantean diferentes tipologías de refuerzo en las familias que se deben considerar: (1) el reforzamiento positivo, que hace referencia a una conducta que se debe repetir mediante respuestas reiteradas positivas o reforzadores verbales, materiales, físicos. Se fundamenta en el estímulo no despectivo; (2) el reforzamiento negativo consiste en quitar un estímulo negativo, algo que no sea apetitivo para el individuo, lo cual ayuda a reforzar una conducta deseada, lo que se pretende es que dicha conducta se vuelva a repetir (Byiers et al., 2014).

Desde la psicología, los refuerzos se consideran una estrategia útil para constituir nuevos tipos de conducta, de modo que trae beneficios para la convivencia familiar, social o educativa, lo cual favorece la adaptabilidad y la cohesión grupal. Además, el castigo también es considerado una técnica psicológica que genera un proceso de aprendizaje, pero, en comparación con el refuerzo positivo, permite eliminar conductas no deseadas o inapropiadas, como conductas agresivas o comunicación no adecuada, que se busca corregir y eliminar para establecer una conducta alternativa.

De la misma manera, también se presentan dos tipologías de castigos: (1) el castigo positivo consiste en atribuir un tipo de consecuencia con el fin de eliminar la conducta no deseada, así se le proporciona al individuo un estímulo que no sea agradable para disminuir la conducta no deseada; (2) el castigo negativo consiste en retirar el estímulo apetitivo o de agrado en su comportamiento (Amaíz \& Flores, 2018). 
Lo anterior hace referencia, por una parte, a un estímulo que condiciona nuestro aprendizaje y conducta al mismo tiempo por temor a la consecuencia que pueda tener, y, por otro, a cómo el individuo aprende a diferenciar entre lo que se debe hacer y lo que no. Precisamente, este proceso sirve para cambiar una conducta no deseada o no aceptada, de manera que es aquí donde el desarrollo ético se genera en edades tempranas con el aprendizaje ético. Esto tendrá una repercusión en la relación del individuo con la sociedad, ya que la infancia se considera el periodo en el cual el aprendizaje se estimula y fortalece, además de que es cuando se potencian habilidades y competencias que a su vez tienen un impacto para la vida en sociedad.

Del mismo modo, los psicólogos del desarrollo humano dejaron un legado basado en el desarrollo moral del individuo que tiene lugar en edades tempranas. De acuerdo con Colón (2004), Jean Piaget formuló el concepto de moralidad en dos etapas, las cuales denominó heterónoma (comunidad) y autónoma (individual). Este autor afirma que la moralidad se desarrolla gradualmente, lo cual quiere decir que es un proceso de formación conjunta que va de la mano con el desarrollo cognoscitivo del niño: a mayores conocimientos y vivencias, se tiene una mayor certeza de la moralidad y con esta se estructura una ética. Por esto último, la educación en temas humanísticos es fundamental en edades tempranas y debe ser reforzada permanentemente para generar cambios significativos.

Piaget (1974) explica la etapa heterónoma como un momento en el que se aportan juicios simples y rígidos, en donde no se logran identificar las diversas alternativas y se actúa de manera egocéntrica sin pensar en las consecuencias, pues se considera que las reglas no se pueden modificar. En contraste, en la segunda etapa, la autónoma, que se caracteriza por la madurez cognitiva del niño a medida que el proceso ocurre, el niño tiende a ser menos egocéntrico, ya que interactúa con los demás y su percepción rígida cambia. Se considera que las reglas establecidas pueden cambiar, es por esto que al principio los niños únicamente se limitan a obedecer normas que establecen los padres, mientras que más adelante aceptan las reglas aun cuando no se sientan identificados, por ello se reorganizan según su beneficio y pensando en el conjunto con la sociedad (Piaget, 1974). 
A partir de lo anterior, Kohlberg (1981; citado por Colón, 2004), afirma que en las edades tempranas se busca un cierto tipo de aprobación, es por esto que el individuo tiende a mantener buenas relaciones con su entorno, pero en edades avanzadas se acopla y acepta voluntariamente aquellas normas éticas de la sociedad o en ocasiones puede llegar a hacer algunas excepciones dependiendo del contexto en el que se encuentre. Es así que el núcleo familiar, educativo, social o laboral en el que se tiende a comportar de manera diferente evidencia el grado de relación y compromiso con que el individuo acoge las reglas y normas fundamentales. Como consecuencia, la persona tiende a obedecer a una moralidad conforme su conciencia individual. Asimismo, en etapas avanzadas busca principios morales más amplios, más allá de los valores y principios de la sociedad.

Para Colón (2004), el comportamiento moral tiene una variante a partir del género, ya que los hombres determinan su comportamiento moral en servicio de los principios generales de justicia y de equidad, a diferencia de las mujeres, cuyo comportamiento moral se rige por la responsabilidad hacia su prójimo, basado en sacrificios por la ayuda constante. El género femenino está orientado constantemente a las relaciones y el bienestar individual, el cual se da en tres etapas clave: la primera se basa en la supervivencia y la segunda en el sacrificio de sus deseos por el bienestar de los demás, mientras que en la tercera el desarrollo moral está en desacuerdo con la violencia. En todo caso, se evita todo tipo de violencia que haga daño a la vida o integridad del ser humano. Pese a esto, se debe resaltar que las construcciones en torno al género varían conforme a las sociedades, de manera que unos postulados pueden variar según el contexto social, las creencias, las tradiciones y los principios morales.

Asimismo, es necesario mencionar el impacto que tiene la familia en relación con la ética y la moral, dado que tiene un significado importante para la sociedad. Conviene subrayar que la familia es la forma primaria de organización humana y, habría que decir también, parte de un grupo de referencia para el individuo, sin importar cómo esté compuesta. Los miembros son encargados de establecer vínculos de relación humana, en vista que es allí donde se genera el aprendizaje social, ético y moral a través de vínculos de afecto (Ciurlo, 2014).

Ejemplo de ello, la familia se apropia del lenguaje encargado de la comunicación y la socialización, es aquí donde los niños aprenden a sentir y a pensar, 
y donde reciben las principales pautas sobre los valores. Es por esto que se considera que esta tiene un valor especial y primario en relación con el sistema de relaciones sociales y la formación de la ética, toda vez que mantiene una importancia para los diferentes contextos de la vida humana que vinculan al ser de por vida (Fabelo, 2001).

La familia no se deja permear negativamente, ya que está constantemente inmersa en el mundo. Por consiguiente, la familia es la que forma al individuo para que conviva en sociedad y actúe con base en principios éticos, sobre lo que puede y no debe hacer. En este sentido, se considera que existe una clara relación entre las pautas de crianza en el entorno familiar y la conducta en la sociedad, así como que estas pautas permean a su vez en la ética profesional. Dado que definitivamente los valores que el individuo adquiere a edad temprana forman una estructura en su personalidad, es más difícil que cambie con el tiempo. Por esta razón se considera que si bien la educación y la formación de valores es parte de la responsabilidad que tiene la sociedad, la tarea de los padres y las madres, como formadores y educadores, consiste en proveer las herramientas necesarias para que la persona se enfrente a contextos diferentes y de manera autónoma (Fabelo, 2001).

\section{La conducta en sociedad y la ética profesional}

Cuando se menciona que es importante fortalecer la conducta del individuo a partir de las pautas de crianza que se dan en el entorno familiar en relación con la ética, se hace referencia también a que la familia es un sistema de aprendizaje de fundamentos que forma y transforma la conducta del individuo. Por una parte, dado que la familia es el espacio donde se desarrollan los primeros procesos de interacción y de aprendizaje, es fundamental educar correctamente (desde la moral), ya que es más fácil educar que reeducar ( $\mathrm{S}$. Dasuky, "Cuatro versiones de la ética y la moral" [notas de clase], 2010).

Asimismo, Dasuky afirma que la ética se considera el arte de vivir de una manera adecuada, lo cual trasciende al pensar moral, de tal manera que tiene como objetivo el buen funcionamiento de las distintas áreas de la vida humana en las que se experimentan los valores, las normas y los juicios tradicionales 
(S. Dasuky, "Cuatro versiones de la ética y la moral" [notas de clase], 2010). Todos nacemos y nos formamos dentro de una moral social en la que existe una responsabilidad de convertirnos en seres éticos, es por esto que se resalta la importancia de las primeras pautas de crianza en el entorno familiar. La familia es entonces un sistema de constante relación.

Para Fabelo (2001), la familia preserva una relación con tres dimensiones en la que la concibe como armonía entre la conducta en sociedad, la ética y la moral: (1) la dimensión objetiva hace referencia a que la familia es un valor en sí misma, (2) la dimensión instituida da cuenta de que la familia da principio y establece los valores dentro de sí misma, y (3) la dimensión subjetiva describe la influencia valorativa y mediadora de la familia en relación con la interacción en sociedad. Además, este autor afirma que existe una relación entre las dimensiones que se materializan en la familia.

Por lo anterior, es importante que la crianza de los hijos en el hogar establezca normas y límites claros de lo que está o no permitido. De esta manera, cada individuo del sistema puede actuar de modo responsable y autónomo, lo cual no solo propicia el respeto entre los miembros de dicha conformación familiar, sino que además tiene una influencia en la convivencia y la armonía en el hogar. En contraste, no tener claro los límites ni las normas dentro del hogar genera actos inmorales, pues se pierden los valores de respeto y honestidad, lo cual tiene graves consecuencias.

Por naturaleza, una buena conducta favorece la sana convivencia. Se debe evitar cualquier tipo de mal hábito que se naturalice en conductas que afecten las relaciones sociales, debido a que cualquier valor negativo que se arraigue dentro del núcleo familiar impactará en la consolidación de principios, y si este tampoco es reprendido en la familia, afectará la crianza de los hijos. Parte del éxito de la sana convivencia consiste en la práctica de diversos valores, esto como parte del fortalecimiento del vínculo tanto familiar como social (Febles, 2018).

Al respecto, González y Pulido (2014) señalan que la ausencia de los valores en el contexto familiar es uno de los principales aspectos que afectan el desarrollo de la personalidad de un individuo. Asimismo, explican que existen diversos factores que favorecen la sana convivencia, como un trato afectivo 
entre los miembros y el tiempo entre familia, los cuales ofrecen un aprendizaje mutuo para el desarrollo de competencias para afrontar la vida.

Es por esto que la responsabilidad de los padres frente a la crianza de los hijos se debe impartir desde el concepto de autoridad, ya que si se carece de esta, la crianza no será efectiva porque el individuo (hijo) no tendrá una relación de respeto que evidencie la importancia de la autoridad de los padres, considerada como elemento de control de la conducta negativa y límite de los malos hábitos y principios socialmente negativos, es decir, se trata de un limitante para la conducta negativa en el que tanto madre como padre deben coincidir en etapas tempranas de la formación. Sin embargo, es importante señalar que la autoridad se debe imponer evitando el maltrato físico y verbal, los castigos exagerados, el irrespeto o los sobornos, ya que esto puede traer consecuencias como miedo, odio o irrespeto, lo cual deteriora las relaciones familiares, sociales, la comunicación y la confianza. En consecuencia, el respeto a la autoridad en la edad adulta se materializará en el campo social en conductas como la responsabilidad y en la autonomía moral del individuo, toda vez que diferenciará lo que es moralmente correcto.

Esto no quiere decir que los padres sean los únicos que tienen una responsabilidad en esta educación, pero si el niño está orientado adecuadamente desde el hogar, los valores que ha interiorizado guiarán la adquisición de nuevas normas a su vida en la realidad social externa a la que evoluciona (Fabelo, 2001). Es decir, aunque con dificultad, en el hogar se adquieren cimientos fuertes para que la persona afronte el futuro en diversos contextos, no solo en el familiar. Si los padres de familia no imparten estos valores, se genera una cadena que avanza de generación en generación, ya que estos valores constituyen la base para una sana convivencia y familia.

Según Obregón (2012; citado en Mendoza et al., 2013), de cierta manera los padres tienen la obligación de dar ejemplo de buena conducta (actuar moralmente y no de modo inmoral), ya que esto no solo es determinante en la formación del niño, sino que también permea las relaciones y la convivencia con los otros dentro de este mismo contexto. Asimismo, señala que la escuela es la encargada de fortalecer el concepto de los valores ya formados en el núcleo familiar. 


\section{Familia como valor}

La familia mantiene aún en este tiempo una importancia dentro de la sociedad, pues se considera fundamental para el individuo y como un valor en sí misma, denominada así por su trascendencia y validez, y valorada como parte primaria de la organización del ser humano. Esta también se enmarca en una apropiación de características y del lenguaje familiar, en el cual el individuo aprende a sentir y a ver el mundo, a conocerlo. De igual forma, en la familia se conciben las formas de relacionarse que permiten la supervivencia, esto quiere decir que el individuo aprende a diferenciar entre lo que puede y no puede hacer.

Conviene enfatizar que dentro del núcleo familiar es fundamental que el individuo adquiera normas de conducta y de relación en cuanto a la formación ética y moral. Para él, discernir el comportamiento es un proceso de identificación a través de su medio inmediato familiar, en la que los padres o tutor encargado asumen la responsabilidad de orientar este proceso de conciencia frente a los valores (Fabelo, 2001).

\section{La familia como factor instituyente de valores}

A partir de lo anterior, se entiende a la familia como una forma de organización humana, comprendida en términos de autonomía y con variables. Esto quiere decir que cada núcleo familiar es responsable, autónomo y tiene la capacidad de establecer ciertas normas, las cuales se encargan de regular el comportamiento de cada uno de sus miembros. Aunque dichas normas se basan en los valores, cabe aclarar que cada sistema tiene su propio sistema de creencias individual, de forma que la familia se entiende como núcleo autónomo, un sistema horizontal de autoridad y respeto que establece unas determinadas normas y valores, las cuales son las que aprenden y desarrollan o ejecutan en el ámbito de interacción social (Musitu, 2005).

\section{La familia como mediador de influencias valorativas}

Los valores que la familia instituye tienen diferentes fuentes. Muchos de ellos no son originarios del propio seno familiar, sino procedentes de otros 
ámbitos. Debido precisamente a la significativa presencia que tiene la familia en la formación de los sistemas subjetivos de valores en las primeras etapas de la formación de la personalidad, se constituye en uno de los mediadores fundamentales de todas las influencias valorativas. En este sentido, la familia actúa como especie de intermediario en relación con los factores de naturaleza valorativa que trasladan su influjo hasta cada uno de sus miembros desde la vida, la comunidad, otras instancias educativas, los medios de comunicación masiva, el discurso político, las leyes, entre otros aspectos (Merino, 2007).

Asimismo, la familia proyecta los preceptos morales vigentes en la sociedad y, a través de las tradiciones, los de las generaciones precedentes. Es por estas razones que se puede afirmar que la familia es una especie de termómetro social que reproduce y refleja en qué situación se encuentra la sociedad, a qué sistema socioeconómico pertenece, por dónde anda este, en qué etapa se encuentra. En este sentido, se considera más importante el ejemplo y la práctica de los valores — es decir, de la acción y ejecución de todos los miembros sin excepciones-, que la imposición, conocida como la retórica discursiva. Por lo tanto, es clave ser conscientes del discurso y acompañarlo de la acción constante y cotidiana, de tal manera que sea posible implementar los valores para lograr que el individuo adopte una conducta de respeto igualitario hacia los otros, pues de lo contrario es difícil hacer que adquiera un comportamiento ideal o moral (Fabelo, 2001).

Existe también una preocupación por la crisis de valores. Se trata de un fenómeno que afecta al mundo entero en la actualidad y en el cual el individuo adopta una actitud nihilista: el ser humano no le da valor a ningún tipo de autoridad y hace un cuestionamiento absoluto de todos los valores tradicionales. En la actualidad existe un efecto negativo que está promoviendo el egoísmo, de modo que las personas buscan siempre su beneficio individual y dejan de lado valores como el respeto, la tolerancia, la solidaridad, la equidad, la honestidad, entre otros. De hecho, la sociedad está pensada para el mercado, razón por la cual el lucro individual hace que la persona actúe de forma corrupta e inapropiada, es decir, tenga un comportamiento inmoral relacionado con las ideas arraigadas de triunfo. Por lo tanto, Merino (2007) resalta: 
La verdad es que por ser la familia el tejido fundamental sobre el que se desarrolla la persona y la sociedad, todo cambio que afecta a estas afecta también a la familia y viceversa. En este sentido, los estudios sobre estos cambios coinciden en relacionar la triada persona-familia-sociedad e insisten en la importancia que tiene la educación, ya sea como elemento catalizador de los cambios o como instancia para la orientación positiva y constructiva de ellos. (p. 115)

El problema se suele desarrollar en un ámbito social, pero también se puede dar o trasladar al familiar. Por esta razón, es necesario resaltar la importancia de fortalecer los valores familiares a través de sus mismas dinámicas como herramienta para favorecer la sana convivencia en la familia y la sociedad. En este sentido, en cuanto contexto significativo, la familia debe ser la encargada de promocionar y fortalecer constantemente los valores en el individuo y en las nuevas generaciones, de allí que sea de suma importancia potenciar como sistema insustituible la moral y los valores que impactan al mismo tiempo en el mundo en el escenario contemporáneo.

\section{Entorno familiar para la formación de la ética profesional y su influencia en la conducta del individuo en sociedad}

A lo largo del documento se ha resaltado la importancia del entorno familiar para la formación de la ética y cómo esta forma bases para el comportamiento en sociedad. Desde esta perspectiva, a continuación se aborda el trascendental papel que tiene la ética en el ámbito profesional y su respectivo impacto en esta área de la vida de un ser humano a lo largo de su desarrollo, desde la enseñanza en la familia y el respaldo en las escuelas que tiene como objetivo la aplicación en el ámbito profesional.

\section{La importancia de la educación y la formación}

\section{¿Qué es la educación y la formación?}

La educación y la formación son dos términos similares que tienen un trasfondo conceptual distinto. El primero es entendido como un proceso racional complejo que implica la relación entre el individuo, el entorno y la sociedad. Según Luengo (2004), la educación inicia desde la familia y articula 
otros entornos sociales de interacción social como la escuela y el trabajo. Dicho proceso articula dos componentes importantes: (1) la enseńanza, entendida como la transmisión de conocimientos, ideas, valores, principios y hábitos, y (2) el aprendizaje, entendido como la adquisición y la apropiación de conocimientos, ideas, valores, principios y hábitos. En consecuencia, la educación es un proceso inherente que se genera en el interior de cada uno de los grupos sociales y que implica la adquisición y aplicación de una serie de conocimientos, habilidades y capacidades de manera individual y colectiva.

Por su parte, la formación es considerada un fin que involucra la adquisición de conocimientos, aprendizajes y habilidades de manera perdurable. Según Flórez y Vivas (2007), una condición de la existencia humana es la necesidad de formarse, integrarse y convertirse en un ser espiritualmente capaz, esto mediante el trabajo compartido y la reflexión crítica. Es así que el concepto de formación propone y reconoce que el individuo no solo se moldea con el exterior, sino que también se humaniza y enriquece desde su interior.

\section{¿Cuál es la importancia del educador y formador desde el campo militar?}

La educación, la formación y el entrenamiento son pilares fundamentales de la doctrina militar. Según el Centro de Doctrina del Ejército (Cedoe, 2016a), la doctrina se concibe como los principios fundamentales de las Fuerzas Militares que guían las acciones en apoyo del cumplimiento de los objetivos nacionales. Está compuesta por principios, tácticas, técnicas, procedimientos, términos y símbolos.

Por lo anterior, el Manual de liderazgo 6-22 de la doctrina Damasco establece la importancia y necesidad institucional de educar a líderes militares y civiles mediante procesos tales como ampliar el conocimiento, fortalecer las capacidades interpersonales, analizar y organizar conocimientos, y tener conciencia de la cultura y de sí mismos (CEDoe, 2016a).

\section{El militar como educador y formador}

Las Fuerzas Militares están integradas por servidores públicos que tienen como objetivo la defensa del orden constitucional, la independencia y la sobe- 
ranía nacional, la democracia y la institucionalidad del Estado, por eso tienen un papel importante relacionado con la construcción de Estado y que está orientado a fortalecer la legalidad y la legitimidad. Si bien la Fuerza Pública no es beligerante ni puede ejercer el sufragio, como tampoco intervenir en actividades o debates de partidos o movimientos políticos (Constitución Política, artículo 219, 1991), son ciudadanos que tienen deberes y derechos para con la sociedad, lo cual implica que deben actuar con transparencia, defender los valores institucionales y ser ejemplo social en todos sus ámbitos, incluyendo el familiar.

Así las cosas, las instituciones militares tienen el deber social de formar y educar líderes militares que sean íntegros en todas sus dimensiones (familiar y organizacional), tanto morales como éticas, para que desarrollen su misión constitucional, la cual se caracteriza por el uso de la fuerza en un escenario de conflicto armado y por la construcción de paz mediante el fortalecimiento de la legitimidad y la gobernanza mediante la educación y la formación, el dar y ser ejemplo como edificadoras de paz. Es así que, desde dicha dimensión, un líder militar multidimensional debe tener un comportamiento ético excepcional en el trato que da a su subalterno y al superior, teniendo presente los niveles operacional y estratégico (CEDOE, 2016b).

\section{Formas}

El problema de ética y moral en las sociedades modernas es un efecto de la corrupción que atenta contra la transparencia y la justicia, lo cual ha afectado todos los niveles de las organizaciones. En el caso del Ejército Nacional, la corrupción debe ser contenida desde la formación del militar, debido a que a largo plazo es educador y formador de sus propios hombres y mujeres.

\section{Medidas de prevención}

Uno de los mayores problemas que enfrenta la sociedad colombiana es la corrupción, un fenómeno que amenaza la estabilidad y la seguridad de las instituciones públicas, al tiempo que genera un deterioro de los valores democráticos, éticos y morales del funcionario público. 
De acuerdo con el Manual de generalidades éticas para la vocación militar, del Comando General de las Fuerzas Militares (2016), el militar debe ser un ejemplo de carácter y firmeza. En este sentido, el militar debe actuar desde su ética profesional, es decir, desde la aplicación de principios institucionales y morales adquiridos. De igual modo, desde el enfoque ético, el militar debe ser moral mediante el respeto de los principios, los valores y las virtudes de su vocación. Por tal razón, el militar se convierte en un educador y formador en doble vía, como ser formado en cada institución y como ejemplo de educación (Acosta et al., 2012).

\section{¿Cómo lograrlo?}

Desde un punto de vista crítico, en la academia y en las instituciones públicas existen recursos teóricos y educativos suficientes. El problema puntual consiste en que hace falta que cada uno de los miembros civiles y militares en las Fuerzas interiorice los valores éticos de la institución. En este sentido, desde un enfoque comunicativo funcional del manejo de la información, se deben generar estrategias de comunicación y propaganda en las Fuerzas para infundir la doctrina y prevenir la falta de transparencia institucional y los casos de corrupción, con el objetivo de fortalecer la moral, la ética profesional y la imagen institucional.

Específicamente, la estrategia de prevención debe establecer los siguientes medios: fortalecer la formación académica en los componentes éticos-humanísticos en todos los niveles de las Fuerzas, mantener una fuerte campaña de comunicación y propaganda que infunda doctrina, y establecer mecanismos de prevención y atención de casos que atenten contra los valores institucionales. Se trata de una transformación de la cultura organizacional del rechazo a cualquier conducta negativa.

\section{Ética y vocación}

Según Agudelo (2013), cuando un individuo se vincula al mundo laboral ya se encuentra en una etapa adulta, de modo que ha hecho frente a las dos etapas previas del desarrollo de la moral que plantea Kohlberg (1981; citado 
por Colón, 2004). En ese momento, el ser humano ha desarrollado una moral con base en los principios que son admitidos por una organización y que dan lugar a un conjunto de reglas, normas, valores y derechos, los cuales tienen el visto bueno de un conjunto de personas que pertenecen a una determinada sociedad. La finalidad de estos principios siempre apunta a constituir una organización justa y beneficiosa para cada uno de los miembros, sin excepción alguna. Por definición, este sería el ideal de comportamiento moral que tiene inicios en la familia y el sistema educativo como aliado.

No obstante, actualmente existe una problemática en relación con los conflictos familiares y las falencias del sistema educativo: el ser humano está en constante contacto con actos inmorales y corruptos que involucran una falta total de ética. Esta situación, que se traslada a diversos ámbitos, hace necesario que se creen herramientas para hacerle frente (Thomas \& Matos, 2008).

A lo largo del tiempo, la familia ha estado inmersa en constantes cambios debido a los diferentes periodos históricos de la sociedad, como, entre otros, la pérdida de los roles familiares, falta de autoridad, violencia, separaciones — de modo que el grupo familiar se fragmenta - y relaciones conflictivas en las cuales se evidencia la falta de valores (Bermúdez et al., 2018). Es por esta razón que el desarrollo ético del hombre y la mujer se debe fomentar adecuadamente para el futuro, de manera que tengan la capacidad de tomar decisiones en pro del bienestar de un sistema, mantener una convivencia armoniosa y generar cambios que se sustenten en los valores éticos, iniciando con el respeto por el otro.

Según Santana (1999; citado por Agudelo, 2013), la familia moldea la conciencia individual del ser humano a través del ejemplo, de modo que cada sistema debe tener como objetivo principal reflejar a sus hijos y futuras generaciones el compromiso por hacer lo correcto. Si bien en la sociedad moderna no existe un parámetro estandarizado de enseñanza, algo que sí es cierto es que la manera en la que la institución o familia eduquen al individuo tendrá consecuencias para su futuro, ya que este adopta dicho parámetro para su vida. De esta manera, se forma una asociación con los sistemas alternos de educación para complementar el proceso y formar funcionarios ejemplares (CabreraGarcía et al., 2021). 
Es así que la moral se debe dirigir hacia la igualdad, la equidad y la reciprocidad, es decir, hacia la construcción de una justicia que incluya a todos y que cobre realidad en los actos cotidianos. Así, será posible pasar de un enfoque centrado en el cumplimiento de reglas y convenciones a otro que apunte a desarrollar en los jóvenes una nueva concepción sobre la justicia, la equidad, el cuidado mutuo, los derechos y el bienestar de las personas. Mientras no se formen seres humanos sensibles, capaces de apropiarse de valores para que sean auténticos, se esconderán en prácticas dañinas o, en caso extremo, se volverán seres apáticos y negativos para la sociedad (Sanromán et al., 2015).

Por lo anterior, la ética y la formación profesional en el contexto mundial deben buscar la formación profesional. Esta es la propuesta de una formación integral que comprenda las capacidades y las competencias para acceder al mundo del trabajo, pero también los valores y las actitudes que moldean la personalidad del sujeto y que contribuyen a que logre un desempeño comprometido y eficaz de su profesión, así como un ejercicio responsable de la ciudadanía (González et al., 2014).

Los pilares de la formación integral son dos: la formación en competencias profesionales y la formación ética, los cuales se deben complementar como dos dimensiones que se articulan para preparar al profesional del nuevo milenio. En este marco, la ética profesional se ha situado como un componente dinámico y sustantivo de la formación integral porque asegura una práctica responsable y eficaz al formar el buen uso de las capacidades profesionales, lo cual resulta fundamental para enfrentar y resolver los complejos problemas de la sociedad contemporánea.

\section{Conclusión}

Es importante resaltar el papel que tiene la sociedad en los procesos éticos del ser humano, puesto que actualmente la ética en la sociedad y en el ámbito profesional se ha visto de cierta manera permeada negativamente por valores negativos que pueden tener origen en etapas tempranas de la vida del individuo. Para ello, las instituciones de educación, incluidas las instituciones militares y su doctrina, deben ser fortalecidas para evitar que se presenten casos aislados de falta de transparencia en el desarrollo de las actividades. 
Asimismo, las Fuerzas Militares deben hacer énfasis en procesos éticos con el objetivo de maximizar el impacto positivo en la ejecución de las actividades, ya que dentro de las organizaciones la vida en sociedad y la ética es considerada como un factor transversal a la formación de los individuos. Actualmente existen muchos indicios que permiten afirmar que Colombia se encuentra inmersa constantemente en actos antiéticos, relacionados con la falta de transparencia en la administración pública, el aumento de la violencia, la intolerancia, entre otros aspectos que afectan el tejido social y frente a los cuales las instituciones militares deben ser referentes. Por esto mismo, se debe fomentar la ética dentro del sistema familiar para que potencie la convivencia y las interacciones en el ámbito profesional y social a través de la adquisición de hábitos moralmente aceptados en esta etapa inicial.

A través de los años, la familia ha pasado por cambios en la estructura de sus valores, los cuales se enmarcan dentro de un contexto determinado que resulta cambiante y acondicionado por la construcción de un tejido social que involucra lo público y lo privado. Por ello, los valores entran en un proceso de crisis constante e inevitable. Hoy por hoy, la sociedad necesita educadores y formadores militares que refuercen los valores que le permiten al individuo generar un impacto y acción en la sociedad. Se resalta que la responsabilidad de la formación y la educación recae sobre todo en la familia, toda vez que es el principal escenario de crianza e interacción en comunidad. En este sentido, la familia como principal institución tiene una función social compartida por los educadores e instituciones privadas y públicas.

\section{Referencias}

Acosta Arévalo, C., Arana Ercilla, M., Mendoza Gómez, M., Valencia García, F., \& Muñoz Niño, J. A. (2012). La evaluación de competencias transversales de lectoescritura en el área de Investigación para la formación del profesional de ciencias militares. Revista Cientifica General José María Córdova, 10(10), 259-285. https://doi.org/10.21830/19006586.237

Agudelo, A. (2013). Aporte ético de la familia y el sistema educativo a las organizaciones. Horizontes Pedagógicos, 15(1), 104-116. https://horizontespedagogicos.ibero.edu.co/ article/view/409

Amaíz A., \& Flores, M. (2018). Abordaje interdisciplinario del tabaquismo en la consulta odontológica: Aplicación de técnicas de intervención psicológicas. Odovtos - Revista Internacional de Ciencias Dentales, 20(1), 41-56. 
Bermúdez Quintana, E. M., Valero Londoño, A. N., Díaz Chavarro, B. C., Castrillón Muñoz, E., \& Castrillón Libreros, D. M. (2018). Perpetuación de violencias verbal y psicológicas en las relaciones afectivas y de familia en adolescentes de Cali. Revista Cientifica General José María Córdova, 16(21), 61-80. https://doi.org/10.21830/19006586.312

Byiers, B., Dimian, A., McComas, J., \& Symons, F. (2014). Efectos del refuerzo positivo y negativo en un arreglo de operantes concurrentes sobre el cumplimiento y la conducta problemática. Psychological Research Records, 4(3), 1757-1771.

Cabrera-García, V. E., Rodríguez-Casallas, M. C., Velásquez-Ladino, L. D., \& Garzón-Cruz, Y. T. (2021). Estabilidad marital en matrimonios distanciados por la misionalidad de las Fuerzas Militares de Colombia. Revista Cientifica General José María Córdova, 19(33), 159-177. https://doi.org/10.21830/19006586.710

Centro de Doctrina del Ejército [CEDOE]. (2016a). Manual fundamental de referencia de Ejército 6-22 Liderazgo. Centro de Doctrina del Ejército.

Ciurlo, A. (2014). Género y familia transnacional: Un enfoque teórico para aproximarse a los estudios migratorios. Revista Cientifica General José María Córdova, 12(13), 127-161. https://doi.org/10.21830/19006586.158

Colón, O. (2004). Fundamentos psicológicos de la moral. http://webcache.googleusercontent. com/search?q=cache:FLeRYP6_hzEJ:ponce.inter.edu/nhp/contents/Inter_Ethica/pdf/ fundamentos_psicologicos_de_la_moral.pdf $+\& \mathrm{~cd}=1 \& \mathrm{hl}=\mathrm{es} \& \mathrm{ct}=\mathrm{clnk} \& \mathrm{gl}=\mathrm{co}$

Comando General de las Fuerzas Militares de Colombia. (2016). Manual de generalidades éticas para la vocación militar. https://www.mindefensa.gov.co/irj/go/km/docs/Mindefensa/ Documentos/descargas/Sobre_el_Ministerio/Control_Interno/documentos/manual_ generalidades_eticas_FFMM.pdf

Constitución Política de Colombia. (1991). Congreso de la República.

Fabelo, J. (2001). Los valores y la familia. Docencia, 8(23), 31-37.

Febles, M. (2018). Aproximación al estudio de la vulnerabilidad psicológica: un proyecto de investigación necesario [conferencia]. VIII Convención Intercontinental de Psicología Hominis 2018. Palacio de las Convenciones, La Habana.

Flórez, R., \& Vivas, M. (2007). La formación como principio y fin de la acción pedagógica. Revista de Educación y Pedagogía, 19(57).

González, K., Mortigo, A., \& Berdugo, N. (2014). La configuración de perfiles profesionales en la educación superior y sus implicaciones en el currículo. Revista Cientifica General José Maria Córdova, 12(14), 165-182. https://doi.org/10.21830/19006586.62

González, R., \& Pulido, J. (2014). Fortalecimiento del vínculo afectivo y corresponsabilidad en padres, madres y cuidadores del nivel de sala materna y caminadores del Jardin Infantil La Paz de la Secretaría Distrital de Integración Social [trabajo de grado, Universidad Católica de Colombia].

Kaplan, H. I., \& Sadock's B. J. (1998). Synopsis of psychiatry. Wolters Kluwer.

Kohlberg, L. (1981). The philosophy of moral development. Harpercollins.

Laurence, S., \& Woodward, W. (1996). B. F. Skinner and behaviorism in American culture. Lehigh University Press. 
Luengo, J. (2004). La educación como objeto de conocimiento. El concepto de educación. En Teorías e instituciones contemporáneas de educación (pp. 30-47). Biblioteca Nueva.

Mendoza, B., Alcerro, J., Ramos, M., \& Mendoza, S. (2013). Metodología de investigación cuantitativa. Universidad Pedagógica Nacional "Francisco Morazán”. https://metodologiadeinvestigacioncuantitativa.files.wordpress.com/2013/05/causas-que-generan-lapc3a9rdida-de-valores-en-las-familias.pdf

Merino P. (2007). Educación moral en la familia: Desafíos y oportunidades. Revista de Estudios y Experiencias en Educación, (11), 113-124.

Musitu Ochoa, G. (2005). Socialización familiar y valores de los adolescentes: Un análisis intercultural. Revista Mexicana de Psicología, 22(1), 79-89.

Padilla Cepeda, J. M. (2014). Reseńa: Educación científica y cultura investigativa para la formación del Profesional en Ciencias Militares de Colombia. Revista Cientifica General José Maria Córdova, 12(13), 362-364. https://doi.org/10.21830/19006586.167

Piaget, J. (1974). El criterio moral en el niño. Fontanella.

Sanromán, R., González, I., \& Villa, M. (2015). Los principios éticos y las obligaciones civiles. Boletín Mexicano de Derecho Comparado, 48(142), 313-337.

Santana, D. (1999). La familia y la formación de ciudadanos virtuosos.

Thomas L., \& Matos R. (2008). La educación en valores: Actividades para su fortalecimiento. EduSol, 8(24), 25-31.

Trillo, F. (2018). Condicionamiento operante. Cómo nos va en la vida, importancia de las consecuencias de los actos. En J. Ruiz (ed.), Conductismo. La psicología del verbo (pp. 55-74). Editorial Lulu. https://www.researchgate.net/publication/328676129_ Condicionamiento_operante_Como_nos_va_en_la_vida_La_importancia_de_las_ consecuencias_de_los_actos 